TRANSACTIONS OF THE

AMERICAN MATHEMATICAL SOCIETY

Volume 336, Number 2, April 1993

\title{
THE PRODUCT OF TWO NORMAL INITIALLY $\kappa$-COMPACT SPACES
}

\author{
ERIC K. vAN DOUWEN ${ }^{\dagger}$
}

\begin{abstract}
We prove that it is independent from ZFC that for every cardinal $\kappa$ the following statements are equivalent:

(a) $\kappa$ is singular;

(b) initial $\kappa$-compactness (defined above the introduction) is productive;

(c) initial $\kappa$-compactness is finitely productive; and

(d) the product of two initially $\kappa$-compact normal spaces is initially $\kappa$ compact.

In particular, MA implies that there are two countably compact normal spaces whose product is not countably compact.
\end{abstract}

In our terminology $T_{1}$ implies regular and normal, and $\kappa, \lambda$, and $\mu$ denote infinite cardinals. Recall that a space is called initially $\kappa$-compact if every open cover of cardinality at most $\kappa$ has a finite subcover, and that initial $\omega$ compactness is equivalent to countable compactness, for $T_{1}$-spaces. The meaning of initially $<\kappa$-compact should be clear.

\section{INTRODUCTION}

The Tychonoff Product Theorem asserts that the product of any number of compact spaces is compact. Since a space $X$ is compact iff it is initially $|X|$ compact this suggests that one consider analogous statements for products of initially $\kappa$-compact spaces, for various $\kappa$.

Novák and Teresaka showed, independently, that there exist two initially $\omega$ compact regular (even zero-dimensional) spaces whose product is not initially $\omega$-compact [N, T]. These spaces are not normal, at least under $\mathrm{CH}($ see $\S 3)$. This suggests the natural question, perhaps due to Bandy (cf. [R, Problem B15]), of whether there exists an example in which the two spaces are normal. Our first result is that MA (= Martin's Axiom) implies that this is the case.

1.1. Theorem. Assume MA. There exist two initially <c-compact normal spaces $E_{0}$ and $E_{1}$ such that $E_{0} \times E_{1}$ is not even initially $\omega$-compact. (In fact, even $E_{0} \times E_{1}$ is normal; see $\S 8$.)

Received by the editors November 8, 1989.

1980 Mathematics Subject Classification (1985 Revision). Primary 54B10, 54D15, 54D30; Secondary $02 \mathrm{~K} 05,54 \mathrm{D} 35$.

Key words and phrases. Initially $\kappa$-compact, generalized $\Sigma$-product, countably compact, $\mathrm{GCH}$, normal, MA, product, regular cardinal, compactification.

Research supported by NSF grant MCS 78-09484.

${ }^{\dagger}$ This paper was originally submitted in 1979, and the author had not completed revisions at the time of his death in 1987. The Editorial Board is grateful to Amer Bešlagić for having undertaken the revisions. 
1.2. Corollary. Assume MA. There exist two countably compact normal spaces whose product is not countably compact.

This is the original motivation for this paper. I do not know if MA is essential in 1.2. However, MA is essential for 1.1, as will be shown below. My original proof of this fact used a certain compactness-like property; I am indebted to Jerry Vaughan for identifying this property as initial $\omega_{1}$-compactness; this was the starting point for what follows.

We now turn our attention to the productivity of initial $\kappa$-compactness for $\kappa>\omega$. The following positive result is due to Stephenson and Vaughan [SV, Theorem 1.1]; it was proved earlier by Saks and Stephenson with the additional condition that all factors are regular [SS, Theorem 4.11].

1.3. Theorem. The product of any number of initially $\kappa$-compact spaces is initially $\kappa$-compact provided $\kappa$ is singular and $2^{\lambda}<\kappa$ for all $\lambda<\kappa$.

Saks and Stephenson ask whether [SS, p. 281], or not [SS, p. 287], initial $\kappa$-compactness is productive for (regular) $\kappa>\omega$. Under GCH we answer this question as follows.

1.4. Theorem. Assume GCH. For every regular $\kappa$ there exist two initially $\kappa$ compact normal spaces whose product is not initially $\kappa$-compact.

An obvious corollary to Theorems 1.3 and 1.4 is

1.5. Theorem. Assume GCH. The following are equivalent for each cardinal $\kappa$ :

(a) $\kappa$ is singular,

(b) the product of any number of initially $\kappa$-compact spaces is initially $\kappa$ compact;

(c) the product of two initially $\kappa$-compact spaces is initially $\kappa$-compact; and

(d) the product of two normal initially $\kappa$-compact spaces is initially $\kappa$-compact.

I do not know if Theorem 1.4 can be proved in ZFC. However, the following corollary to Theorem 1.1 shows that Theorem 1.5 is not true in ZFC.

1.6. Theorem. Assume $M A+c>\omega_{\omega}$. There are two initially $\omega_{\omega}$-compact normal spaces whose product is not initially $\omega_{\omega}$-compact.

Note that in light of Theorem 1.5 this also shows that Theorem 1.1 is not true in ZFC (see also Remark 2.7) and that in fact we have shown that the statement that "initial $\kappa$-compactness is productive for all singular $\kappa$ " is independent from ZFC. However, this result would be just another way of saying that GCH is consistent and independent if the condition in Theorem 1.3 is necessary, i.e., if the fact that initial $\kappa$-compactness is productive implies both that $\kappa$ is singular and that $2^{\lambda}<\kappa$ for all $\lambda<\kappa$. This remains an open question.

A trivial but unexpected and interesting corollary to Theorem 1.5 is our last theorem:

1.7. Theorem. Assume GCH. Then for every cardinal $\kappa$ the following implication holds:

$F_{\kappa}$ : if initial $\kappa$-compactness is finitely productive, then it is productive. 
I do not know if this is true in ZFC. To appreciate the difficulty of this question one should note that $F_{\omega}$ is (vacuously) true, and recall that Frolik has given an example of a space $Y$ such that ${ }^{\omega} Y$ is not initially $\omega$-compact, yet ${ }^{n} Y$ is initially $\omega$-compact for all $n<\omega$ [Fr, Theorem B].

I am also indebted to Jerry Vaughan for spotting a number of inaccuracies in the manuscript of this paper.

\section{Preliminaries}

An ordinal is the set of smaller ordinals, and a cardinal is an initial ordinal. If $A$ is a set, the set of functions $A \rightarrow 2$ is denoted by ${ }^{A} 2$ and carries the product topology ( 2 is discrete). For a set $A$ and a cardinal $\nu$ we put as usual

$$
[A]^{\nu}=\{X \subseteq A:|X|=\nu\} ;
$$

$[A]^{\leq \nu}$ and $[A]^{<\nu}$ are defined analogously. Also, for a set $A$ we define

$$
H(A)=\bigcup\left\{F 2: F \in[A]^{<\omega}\right\} ;
$$

note that $\left\{\left\{y \in{ }^{A} 2: x \subseteq y\right\}: x \in H(A)\right\}$ is a base for ${ }^{A} 2$.

If $A$ is a set, an $A$-sequence is a function $f$ with domain $A$, and is denoted by $\left\langle f_{a}: a \in A\right\rangle$.

A space $X$ is called $\kappa$-bounded if every member of $[X]^{\leq \kappa}$ has compact closure [GFW]; we define $<\kappa$-bounded analogously. We remind the reader of the following facts.

2.1. Proposition. (a) The product of any number of $\kappa$-bounded spaces is $\kappa$ bounded [GFW, Lemma 4].

(b) Every $\kappa$-bounded space is initially $\kappa$-compact [GFW, Lemma 3].

(c) Every regular initially $2^{\kappa}$-compact space is $\kappa$-bounded [SS, Theorem 2.1].

(d) If $\kappa$ is singular, then every initially $<\kappa$-compact space is initially $\kappa$ compact, implicit in [SS, proof of Theorem 4.11].

Each cardinal carries the discrete topology. As usual we identify the points of $\beta \kappa$ with free ultrafilters on $\kappa$; in particular, if $\alpha \in \kappa$ then we do not distinguish $\alpha$ from $\{A \subseteq \kappa: \alpha \in A\}$.

We are particularly interested in the open subspace of $\beta \kappa$ consisting of the nonuniform ultrafilters, i.e., in

$$
\mathrm{NU}(\kappa)=\{p \in \beta \kappa: \exists P \in p(|P|<\kappa)\} .
$$

Note that $\mathrm{NU}(\kappa)=\kappa$ if (and only if) $\kappa=\omega$. We define the set of (small) unbounded subsets of $\mathrm{NU}(\kappa)$ by

$$
\mathrm{UB}(\kappa)=\left\{I \in[\mathrm{NU}]^{\mathrm{cf}(\kappa)} \text { : the closure of } I \text { in } \mathrm{NU}(\kappa) \text { is not compact }\right\} .
$$

2.2. Fact. $\mathrm{NU}(\kappa)$ is $<\operatorname{cf}(\kappa)$-bounded but not $\operatorname{cf}(\kappa)$-bounded.

2.3. Fact. For every $A \subseteq \mathrm{NU}(\kappa)$ whose closure in $\mathrm{NU}(\kappa)$ is not compact there is $I \in \mathrm{UB}(\kappa)$ with $I \subseteq A$.

We omit the easy proofs. We will use Fact 2.3 in the construction of the examples, together with the following easy 
2.4. Fact. Assume GCH. Then $|\mathrm{UB}(\kappa)|=\kappa^{+}$, for all $\kappa>\omega$.

Proof. $\left|[\kappa]^{<\kappa}\right| \leq \kappa^{+}$and $|\beta \lambda|=2^{2^{\lambda}} \leq \kappa^{+}$if $\lambda<\kappa$, hence $\kappa \leq|\mathrm{NU}(\kappa)| \leq \kappa^{+}$, under $\mathrm{GCH}$, so $|\mathrm{UB}(\kappa)| \leq \kappa^{+}$.

To see that $|\mathrm{UB}(\kappa)| \geq \kappa^{+}$, let $A=\left\{p_{\alpha}: \alpha<\kappa\right\} \subset \mathrm{NU}(\kappa)$ be such that for each $\omega \leq \alpha<\kappa, p_{\alpha} \in \beta \alpha$ and $\forall P \in p_{\alpha}(|P|=|\alpha|)$. Any unbounded subset of $A$ of size $\operatorname{cf}(\kappa)$ is in $\mathrm{UB}(\kappa)$, ands there are $\kappa^{\mathrm{cf}(\kappa)}=\kappa^{+}$many of them.

2.5. Remark. Actually one can easily show that $|\mathrm{NU}(\omega)|=\omega$, and, if $\kappa>\omega$, then $|\mathrm{NU}(\kappa)|=\kappa^{\kappa} \cdot \sum_{\lambda<\kappa} 2^{2^{\lambda}}$, in ZFC.

The proof of our final piece of preliminary information is analogous to the proof of the special case $b \kappa=\beta \kappa$, hence is omitted.

2.6. Fact. Let $b \kappa$ be any compactification of $\kappa$ with $\mathrm{NU}(\kappa) \subseteq b \kappa$. If $K$ is a clopen (= closed and open) subset of $b \kappa-\mathrm{NU}(\kappa)$, then there is a $A \subseteq \kappa$, unique up to a set of cardinality $<\kappa$, such that $\bar{A}-\mathrm{NU}(\kappa)=K$ and $\overline{\bar{A}}$ is clopen in $b \kappa$.

(Here "unique up to a set of cardinality $<\kappa$ " means that $\bar{B}-\mathrm{NU}(\kappa)=K$ iff $|(A-B) \cup(B-A)|<\kappa$, for all $B \subseteq \kappa$.

2.7. Remark. In the introduction we gave one proof that Theorem 1.1 requires MA. Here we give another: It is consistent with ZFC that $c$ be singular [Ku]. But if $\mathfrak{c}$ is singular then the product of any number of initially $<\mathfrak{c}$-compact regular spaces is countably compact, as one can easily prove from Proposition 2.1.

These two proofs require $c>\omega_{\omega}$. One can also show that Theorem 1.1 requires MA even if $\mathfrak{c}=\omega_{2}$. Indeed, it is consistent with $\mathrm{ZFC}+\mathfrak{c}=\omega_{2}$ that the product of any number of initially $\omega_{1}$-compact spaces be countably compact [vD, Theorem 7.3].

\section{The BASIC IDEA. I}

In this section we explain the basic idea of how to get two countably compact normal spaces whose product is not countably compact.

Novák constructs two countably compact spaces $X_{i}$ with $\omega \subset X_{i} \subset \beta \omega(\subset$ denotes proper inclusion) for $i<2$ such that

(0) $X_{0} \cap X_{1}=\omega$

Then $X_{0} \times X_{1}$ is not countably compact since $\{\langle n, n\rangle: n<\omega\}$ is a countable subset of $X_{0} \times X_{1}$ which is closed in $X_{0} \times X_{1}$, being the intersection of $X_{0} \times X_{1}$ and the diagonal $\{\langle x, x\rangle: x \in \beta \omega\}$ of the Hausdorff space $\beta \omega$. Under $\mathrm{CH}$ the $X_{i}$ 's are not normal, for otherwise $X_{i}-\omega$ would be $C^{*}$-embedded in $X_{i}$, hence in $\beta X_{i}=\beta \omega$, hence in $\beta \omega-\omega$; also $X_{i}-\omega$ is a proper subset of $\beta \omega-\omega$ which is dense in $\beta \omega-\omega$ since $X_{i}$ is countably compact. But under $\mathrm{CH}$ there are no dense proper $C^{*}$-embedded subspaces in $\beta \omega-\omega$ [FG, Theorem 4.6]. So $\beta \omega$ is the wrong compactification of $\omega$ to use.

We plan to construct a different compactification $b \omega$ of $\omega$ and two suitable subspaces $\Sigma_{0}$ and $\Sigma_{1}$ of $b \omega-\omega$ such that

(1) $\Sigma_{0} \cap \Sigma_{1}=\varnothing$.

Then our examples will be the subspaces

$$
E_{i}=\omega \cup \Sigma_{i} \quad(i<2)
$$


of $b \omega$. Then we get the fact that $E_{0} \times E_{1}$ is not countably compact for free, for $E_{0} \cap E_{1}=\omega$, hence $E_{0} \times E_{1}$ is not countably compact, as above.

We now investigate the question of how one can ensure that $E_{0}$ and $E_{1}$ are countably compact and normal. We first consider countable compactness. The easy proofs of the following fact and its corollary are omitted.

3.1. Fact. Both $E_{i}$ 's are countably compact if and only if

(2) both $\Sigma_{i}$ 's are countably compact; and

(3) $\left.\forall i<2 \forall I \in[\omega]^{\omega}\left(\bar{I} \cap \Sigma_{i}\right) \neq \varnothing\right)$.

3.2. Corollary. If both $E_{i}$ 's are countably compact, then

(4) both $\Sigma_{i}$ 's are dense in $b \omega-\omega$.

We next consider normality. Since disjoint closed sets of any space have disjoint neighborhoods iff their boundaries have disjoint neighborhoods, a moment's reflection shows that the following holds.

3.3. Fact. A sufficient condition for the $E_{i}$ 's to be normal is

(5) every two disjoint closed sets in $\Sigma_{i}$ have disjoint closures in $b \omega-\omega$ $(i<2)$.

Note that this only involves the way the $\Sigma_{i}$ 's sit inside $b \omega-\omega$, and not the way the $\Sigma_{i}$ 's are attached to $\omega$ (cf. (3)).

3.4. Remark. Note that (5) implies that the $\Sigma_{i}$ 's are normal, and if (4) holds, then (5) holds iff the $\Sigma_{i}$ 's are normal and $\beta \Sigma_{i}=b \omega-\omega(i<2)$.

So if we can ensure that (2), (3), and (5) hold, then our $E_{i}$ 's are countably compact and normal. We do not want to have to consider three conditions simultaneously. Therefore the first step of the construction is to pick a compact space $P$ having subspaces $\Sigma_{0}$ and $\Sigma_{1}$ such that (2) and the analogues of (4) and (5), with $P$ instead of $b \omega-\omega$, hold. The second step is to show that $\omega$ has a compactification $b \omega$ with $b \omega-\omega=P$ such that (3) holds. (Note that we need (4) since it is a necessary condition for (3) to hold.) It is just a happy coincidence that $E_{0}$ and $E_{1}$ are not only countably compact but even initially $<$ c-compact.

My first result was that this plan works (but that one needs MA in the second step). This has been the key to the discovery of Theorem 1.4, whose basic idea will be explained in the next section. One does not have to know yet how we perform our two steps. However, it may be helpful to know that there is a purely set-theoretic translation of the existence of $b \omega$, so that all one has to do in the second step is to construct a certain family of subsets of $\omega$.

\section{THE BASIC IDEA. II}

Let $\kappa$ be a regular cardinal. Assuming enough additional axioms we will construct a compactification $b \kappa$ of $\kappa$ and initially $\kappa$-compact subspaces $E_{0}$ and $E_{1}$ such that $E_{0} \times E_{1}$ is not initially $\kappa$-compact .

As before, we ensure that $E_{0} \times E_{1}$ is not initially $\kappa$-compact by making $E_{0} \cap E_{1}$ not initially compact. (This is why $E_{0}$ and $E_{1}$ must be subspaces of the same Hausdorff space.) We cannot have $E_{0} \cap E_{1}=\kappa$, unless $\kappa=\omega$. For we will have to assume that $2^{\lambda} \leq \kappa$ for $\lambda<\kappa$, hence $E_{0}$ and $E_{1}$ are $<\kappa$-bounded by $2.1(\mathrm{c})$, hence so is $E_{0} \times E_{1}$, by $2.1(\mathrm{a})$. 
We choose $E_{0} \times E_{1}$ before we begin the construction. Since we are interested in a $b \kappa$ whose existence is equivalent to the existence of some family of subsets of $\kappa$ (like in the special case $\kappa=\omega$ ), we choose $E_{0} \cap E_{1}=\mathrm{NU}(\kappa)$. Note that this agrees with our choice for $\kappa=\omega$.

So $b \kappa$ is a compactification of $\mathrm{NU}(\kappa)$ as well. We must have suitable subspaces $\Sigma_{0}$ and $\Sigma_{1}$ of $b \kappa-\mathrm{NU}(\kappa)$, then our examples will be the subspaces

$$
E_{i}=\mathrm{NU}(\kappa) \cup \Sigma_{i} \quad(i<2)
$$

of $b \kappa$. (It will not be necessary to give the $E_{i}$ 's and $\Sigma_{i}$ 's the subscript $\kappa$.)

We now investigate when our examples are initially $\lambda$-compact; the reason for considering the cases $\lambda \neq \kappa$ is that in Theorem 1.1 we consider initial $<\mathfrak{c}$-compactness, and because of Remark 7.2.

4.1. Fact. Let $\lambda \geq \kappa$, then the following are equivalent:

(a) Both $E_{0}$ and $E_{1}$ are initially $\lambda$-compact.

(b) The following two statements hold:

(1) both $\Sigma_{0}$ and $\Sigma_{1}$ are initially $\lambda$-compact; and

(2) $\forall i<2 \forall I \in \mathrm{UB}(\kappa)\left(\bar{I} \cap \Sigma_{i} \neq \varnothing\right)$.

Proof. That (a) implies (b) is easy to see. (The assumption $\lambda \geq \operatorname{cf}(\kappa)$ is used to get (2) from (a).) To prove that (b) implies (a) let $i<2$ and let $\mathscr{U}$ be an open cover of $E_{i}$ with $|\mathscr{U}| \leq \lambda$. There is a finite $\mathscr{V} \subseteq \mathscr{U}$ such that $\Sigma_{i} \subseteq \bigcup \mathscr{V}$. There is no $I \subset \mathrm{UB}(\kappa)$ with $I \subseteq \mathrm{NU}(\kappa)-\bigcup \mathscr{V}$, hence by Fact 2.3 the subspace $\mathrm{NU}(\kappa)-\bigcup \mathscr{V}$ is compact. Hence $\mathscr{U}$ has a finite subcover.

The following fact is similar to Fact 3.3, but the proof is slightly less trivial since $E_{i}-\Sigma_{i}$ is no longer discrete if $\kappa>\omega$.

4.2. Fact. A sufficient condition for the $E_{i}$ 's to be normal is that (2) and the following statement hold:

(3) every two disjoint closed sets in $\Sigma_{i}$ have disjoint closures in $b \kappa-\mathrm{NU}(\kappa)$ $(i<2)$.

Proof. Fix $i<2$. We prove that $E_{i}$ is normal by proving

$(\alpha)$ any two disjoint closed subsets of $E_{i}$ have disjoint closures in $b \kappa$.

To see this observe that (2) implies that for any $A \subset \mathrm{NU}(\kappa), \bar{A}-\mathrm{NU}(\kappa)=$ $\overline{\left(\bar{A} \cap \Sigma_{i}\right)}$, where the overbar is the closure operator in $b \kappa$. (Use Fact 2.3 here.) So if $F$ is a closed subset of $E_{i}$,

$$
\bar{F}=\overline{\left(F \cap \Sigma_{i}\right)} \cup(F \cap \mathrm{NU}(\kappa)) .
$$

4.3. Remark. We just showed that (2) and (3) imply $(\alpha)$, or, equivalently, that the $E_{i}$ 's are normal and that $\beta E_{i}=b \kappa(i<2)$. It is easy to see that conversely $(\alpha)$ implies (2) and (3).

\section{OUR CHOICE FOR $b \kappa-\mathrm{NU}(\kappa)$ AND THE $\Sigma_{i}$ 'S}

In the previous two sections we have seen that, given a regular $\kappa$, we need a compact space $P$, having subspaces $\Sigma_{0}$ and $\Sigma_{1}$, such that

(1) $\Sigma_{0} \cap \Sigma_{1}=\varnothing$,

(2) $\Sigma_{i}$ is initially $\kappa$-compact $(i<2)$,

(3) $\Sigma_{i}$ is dense in $P(i<2)$,

(4) disjoint closed subsets of $\Sigma_{i}$ have disjoint closures in $P(i<2)$. 
(Without condition (1) this is trivial.) In all real examples that I am aware of $P$ is a product of compact spaces and the $\Sigma_{i}$ 's are generalized $\Sigma$-products. We will consider the case where $P$ is a product of 2-point spaces: Given a (regular) $\kappa$, we plan to have

$$
b \kappa-\mathrm{NU}(\kappa)={ }^{\left({ }_{2} \kappa\right)} 2
$$

and

$$
\Sigma_{i}=\left\{x \in{ }^{\left(2^{\kappa}\right)} 2:\left|\left\{\alpha<2^{\kappa}: x(\alpha) \neq i\right\}\right|<2^{\kappa}\right\} .
$$

It is obvious that (1) and (3) hold. It follows from the discussion below that (2) and (4) hold, and that in fact

$\left(2^{\prime}\right) \Sigma_{i}$ is initially $<\operatorname{cf}\left(2^{\kappa}\right)$-compact.

The discussion is somewhat more general than is really needed since this is easier.

5.1. Convention. For the remainder of this section $\lambda$ and $\mu$ are cardinals with $\omega<\lambda \leq \mu$, and $\Sigma$ is the subspace

$$
\Sigma=\left\{x \in{ }^{\mu} 2:|\{\alpha<\mu: x(\alpha) \neq 0\}|<\lambda\right\}
$$

of ${ }^{\mu} 2$, and for $A \subseteq \mu$ we identify ${ }^{A} 2$ in the natural way with

$$
\left\{x \in \in^{\mu} 2:\{\alpha<\mu: x(\alpha) \neq 0\} \subseteq A\right\} .
$$

5.2. Proposition. (a) $\Sigma$ is $<\operatorname{cf}(\lambda)$-bounded (hence is initially $<\operatorname{cf}(\lambda)$-compact).

(b) $\Sigma$ is not initially $\operatorname{cf}(\lambda)$-compact.

Proof. If $X \subseteq \Sigma$ has cardinality $<\operatorname{cf}(\lambda)$, then

$$
A=\{\alpha<\mu: x(\alpha) \neq 0 \text { for some } x \in X\}
$$

has cardinality less than $\lambda$, hence ${ }^{A} 2 \subseteq \Sigma$. Since $X \subseteq{ }^{A} 2$, it follows that $X$ has compact closure. This proves (a).

Next let $C$ be a cofinal subset of $\lambda$ with $|C|=\operatorname{cf}(\lambda)$. Then

$$
\{\{x \in \Sigma: x(\alpha)=0 \text { for some } \alpha<\gamma\}: \gamma \in C\}
$$

is an open cover of $\Sigma$ which has no finite subcover. This proves (b).

5.3. Theorem. If $\lambda$ is regular, then every two disjoint closed subsets of $\Sigma$ have disjoint closures in ${ }^{\mu} 2$.

Proof. This follows from the proofs of [C, Theorem 1; Ko, Theorem 1; KoM, Basic Lemma]. We sketch the proof for the convenience of the reader.

For $x \in \mu_{2}$ let $\operatorname{supt}(x)=\{\alpha \in \mu: x(\alpha)=1\}$. Assume that $H, K \subset \Sigma$ and that $p \in{ }^{\mu_{2}}$ is such that $p \in \bar{H} \cap \bar{K}$. By recursion, construct in $\omega$ steps a set $A \subset \mu$ with $|A|<\lambda$ so that for every neighborhood $U$ of $p \mid A$ in ${ }^{A} 2$ there are points $h \in H$ and $k \in K$ with $\operatorname{supt}(h) \cup \operatorname{supt}(k) \subset A$ and $\{h\lceil A, k \uparrow A\} \subset U$.

Then $q \in{ }^{\mu} 2$, defined by $q|A=p| A$ and $\forall \alpha \in \mu-A(q(\alpha)=0)$, is in $\Sigma$ and $q \in \bar{H} \cap \bar{K}$.

5.4. Remark. Instead of $\Sigma$ one can consider

$$
\left\{x \in{ }^{\mu} 2:|\{\alpha<\mu: x(\alpha) \neq p(\alpha)\}|<\lambda\right\}
$$

for any $p \in \epsilon^{\mu} 2$. 
5.5. Remark. $\Sigma$ is not normal if $\operatorname{cf} \lambda=\omega$. For then $\Sigma$ is not countably compact by $5.2(\mathrm{~b})$, but is pseudocompact since it has the dense subspace

$$
\left\{x \in{ }^{\mu} 2:|\{\alpha<\mu: x(\alpha) \neq 0\}|<\omega_{1}\right\},
$$

which is countably compact by $5.2(\mathrm{a})$. I did not investigate whether or not $\Sigma$ is normal if $\omega<\operatorname{cf}(\lambda)<\lambda$.

We need GCH (or MA if $\kappa=\omega$ ) not only to construct our spaces, but also to prove that $E_{0}$ and $E_{1}$ are normal since each $2^{\kappa}$ is (trivially) regular under $\mathrm{GCH}$, and $2^{\omega}$ is regular under MA. The latter fact also implies that $E_{0}$ and $E_{1}$ are initially $<\mathfrak{c}$-compact if $\kappa-\omega$.

\section{A SET-THEORETIC TRANSLATION}

We are interested in finding a compactification $b \kappa$ of $\kappa$ such that

(1) $\mathrm{NU}(\kappa) \subseteq b \kappa$ and $b \kappa-\mathrm{NU}(\kappa)={ }^{\left(2^{\kappa}\right)} 2$; and

(2) $\forall i<2 \forall I \in \mathrm{UB}(\kappa)\left(\bar{I} \cap \Sigma_{i} \neq \varnothing\right)$.

It will be convenient to have a simple set-theoretic translation of the existence of such a compactification. The following notation is useful to cut down on the length of the formulas. Recall that $H(\alpha)=\bigcup\left\{F^{2}: F \in[\alpha]^{<\omega}\right\}$.

6.1. Notation. If $\left\langle X_{\xi}: \xi<\alpha\right\rangle$ is an $\alpha$-sequence of subsets of a set $S$, then for every $f \in H(\alpha)$ we define $X(S, f) \subseteq S$ by

$$
X(S, f)=\left\{s \in S: \forall \xi \in \operatorname{dom}(f)\left(s \in X_{\xi} \Leftrightarrow f(\xi)=1\right)\right\},
$$

or, equivalently,

$X(S, f)=\bigcap\left\{X_{\xi}: \xi \in \operatorname{dom}(f) \wedge f(\xi)=1\right\} \cap \bigcap\left\{S-X_{\xi}: \xi \in \operatorname{dom}(f) \wedge f(\xi)=0\right\}$

if $f \neq \varnothing$, and $X(S, \varnothing)=S$.

6.2. Lemma. The following statements are equivalent for a regular cardinal $\kappa$ :

(a) $\kappa$ has a compactification $b \kappa$ for which (1) and (2) holds; and

(b) there is a $2^{\kappa}$-sequence $\left\langle X_{\xi}: \xi<2^{\kappa}\right\rangle$ of subsets of $\kappa$ such that

(3) $\forall f \in H\left(2^{\kappa}\right)[|X(\kappa, f)|=\kappa]$; and

(4) $\forall i<2 \forall I \in \mathrm{UB}(\kappa) \exists x \in \Sigma_{i} \forall f \in H\left(2^{\kappa}\right)[f \subseteq x \Rightarrow \forall \eta<\kappa \exists y \in$ $I(X(\kappa, f)-\eta \in y)]$.

Proof. We only need the implication (b) $\rightarrow$ (a), but the proof of the reverse implication is felt to help in understanding what is going on.

We first show how a compactification $b \kappa$ of $\kappa$ satisfying (1) gives rise to a $2^{\kappa}$-sequence $\left\langle X_{\xi}: \xi<2^{\kappa}\right\rangle$ of subsets of $\kappa$ satisfying (3) and conversely. Once we are able to switch back and forth it is easy to see that (2) and (4) are mere translations of each other.

Remember that $\mathrm{NU}(\kappa)$ is $<\kappa$-bounded since $\kappa$ is regular, by 2.2 .

For $\xi<2^{\kappa}$ define the subbasic clopen set $K_{\xi} \subseteq\left(2^{\kappa}\right) 2$ by

$$
K_{\xi}=\left\{x \in{ }^{\left(2^{\kappa}\right)} 2: x(\xi)=1\right\} .
$$

And for $f \in H\left({ }^{\left(2^{\kappa}\right)} 2\right)$, let $K\left({ }^{\left(2^{\kappa}\right)} 2, f\right)=\left\{x \in{ }^{\left(2^{\kappa}\right)} 2: f \subseteq x\right\}$.

Assume $b \kappa$ is a compactification of $\kappa$ satisfying (1). By Fact 2.6 there is for each $\xi<2^{\kappa}$ an $X_{\xi} \subseteq \kappa$, unique up to a set of cardinality less than $\kappa$, such that 
(5) $\bar{X}_{\xi} \cap{ }^{\left(2^{\kappa}\right)} 2=K_{\xi}$, and $\bar{X}_{\xi}$ is clopen.

For every $f \in H\left(2^{\kappa}\right)$ we have $X(\kappa, f)^{-} \cap{ }^{\left(2^{\kappa}\right)} 2=K\left({ }^{\left(2^{\kappa}\right)} 2, f\right) \neq \varnothing$, hence $|X(\kappa, f)|=\kappa$ since $\mathrm{NU}(\kappa)$ is $<\kappa$-bounded. This proves (3). Observe that

(6) for every $x \in{ }^{\left(2^{\kappa}\right)} 2$ the family

$$
\left\{(X(\kappa, f)-A)^{-}: f \in H\left(2^{\kappa}\right) \wedge f \subseteq x \wedge A \in[\kappa]^{<\kappa}\right\}
$$

is a neighborhood base for $x$ in $b \kappa$.

Next assume a $2^{\kappa}$-sequence $\left\langle X_{\xi}: \xi<2^{\kappa}\right\rangle$ of subsets of $\kappa$ is given that satisfies (3). We let $b \kappa$ be the smallest compactification of $\kappa$ for which every member of

$$
\mathscr{A}=\left\{X_{\xi}: \xi<2^{\kappa}\right\} \cup[\kappa]^{<\kappa}
$$

has open closure in $b \kappa$. The best way to think of $b \kappa$ is as follows: let $\mathscr{B}$ be the subalgebra of $\mathscr{P}(\kappa)$, the field of all subsets of $\kappa$, generated by $\mathscr{A}$, and let $b \kappa$ be the Stone space of $\kappa$. Recall that points of $b \kappa$ are ultrafilters in $\mathscr{B}$, so if we identify $\alpha \in \kappa$ with $\{B \in \mathscr{B}: \alpha \in B\}$ (as we did when we identified $\beta \kappa$ with the space of all ultrafilters on $\kappa$ ), then $b \kappa$ becomes a compactification of $\kappa$. With a similar identification one gets $\mathrm{NU}(\kappa) \subseteq b \kappa$. It remains to show that there is a natural homeomorphism $h:{ }^{\left({ }^{\kappa}\right)} 2 \rightarrow(b \kappa-\mathrm{NU}(\kappa))$. One can define a function $h:{ }^{\left(2^{\kappa}\right)} 2 \rightarrow b \kappa$ by

$$
h(x)=\left\{X(\kappa, f)-A: f \in H\left(2^{\kappa}\right) \wedge f \subseteq x \wedge A \in[\kappa]^{<\kappa}\right\} .
$$

It is easy to see, using (3), that this is well defined, i.e., that each $h(x)$ is an ultrafilter of $\mathscr{B}$, and that $h$ is a homeomorphism of ${ }^{\left(2^{\kappa}\right)} 2$ onto $b \kappa-\mathrm{NU}(\kappa)$. So we will from now on identify $b \kappa-\mathrm{NU}(\kappa)$ with ${ }^{\left(2^{\kappa}\right)} 2$. Note that our definition of $h$ tells us that (5) and (6) hold for the $b \kappa$ we constructed from the $X_{\xi}$ 's.

In summary, given $b \kappa$ we choose the $X_{\xi}$ 's such that (5) holds. In turn the $X_{\xi}$ 's uniquely determine $b \kappa$; this is where we used the fact that each $X_{\xi}$ is unique up to a set of cardinality less than $\kappa$. This completes the description of the interrelation of $b \kappa$ and the $X_{\xi}$ 's. It remains to show that (2) and (4) are equivalent.

Let $i<2, I \in \mathrm{UB}(\kappa)$, and $x \in \Sigma_{i}$ be given. It follows from (5) that

$$
x \in \bar{I} \text { iff } \forall f \in H\left(2^{\kappa}\right) \forall A \in[\kappa]^{<\kappa}\left(f \subseteq x \Rightarrow\left((X(\kappa, f)-A)^{-} \cap I \neq \varnothing\right)\right) \text {, }
$$

hence, since $\kappa$ is regular,

$$
x \in \bar{I} \text { iff } \forall f \in H\left(2^{\kappa}\right)\left(f \subseteq x \Rightarrow(\forall \eta<\kappa)\left((X(\kappa, f)-\eta)^{-} \cap I \neq \varnothing\right)\right) .
$$

But if $y \in \mathrm{NU}(\kappa)$ and $T \subseteq \kappa$, then $y \in \bar{T}$ iff $T \in y$ since $\mathrm{NU}(\kappa)$ consists of ultrafilters on $\kappa$. This proves that (2) and (4) are equivalent, as required.

\section{THE CONSTRUCTION}

Let $\kappa$ be a regular cardinal, $\kappa \geq \omega$. We want to construct a certain $2^{\kappa}$ sequence $\left\langle X_{\xi}: \xi<2^{\kappa}\right\rangle$ of subsets of $\kappa$. We change Notation 6.1 by writing $X(f)$ instead of $X(\kappa, f)$. Recall that we want our $X_{\xi}$ 's to satisfy

(1) $\forall f \in H\left(2^{\kappa}\right)[|X(f)|=\kappa]$; and

(2) $\forall i<2 \forall I \in \mathrm{UB}(\kappa) \exists x \in \Sigma_{i} \forall f \in H\left(2^{\kappa}\right)[f \subseteq x \Rightarrow \forall \eta<\kappa \exists y \in$ $I(X(f)-\eta \in y)]$. 
We assume that $\mathrm{GCH}$ holds, or that MA holds if $\kappa=\omega$. In both cases $|\mathrm{UB}(\kappa)|=2^{\kappa}$ by Fact 2.4 . Hence we can list $\mathrm{UB}(\kappa)$ as $\left\langle I_{\xi}: \xi<2^{\kappa}\right\rangle$.

With transfinite recursion on $\xi<2^{\kappa}$ we will construct $x_{i, \xi} \in \Sigma_{i}(i<2)$ and $X_{\xi} \subseteq \kappa$. We will be interested in the following conditions:

$\left(\mathrm{A}_{\alpha}\right) \forall f \in H(\alpha)[|X(f)|=\kappa]$; and

$\left(\mathrm{B}_{\alpha}\right) \forall i<2 \forall \xi<\alpha \forall f \in H(\alpha)\left[f \subseteq x_{i, \xi} \Rightarrow \forall \eta<\kappa \exists y \in I_{\xi}(X(f)-\eta \in y)\right]$.

Let $\beta \leq 2^{\kappa}$, and assume $x_{i, \xi}(i<2)$ and $X_{\xi}$ to be constructed for all $\xi<\beta$ in such a way that $\left(\mathrm{A}_{\alpha+1}\right)$ and $\left(\mathrm{B}_{\alpha+1}\right)$ hold for all $\alpha<\beta$. Then $\left(\mathrm{A}_{\beta}\right)$ and $\left(B_{\beta}\right)$ hold. So if $\beta=2^{\kappa}$, then our construction is complete, and (1) and (2) hold. Now assume $\beta<2^{\kappa}$.

Claim. There is $u \in{ }^{\beta} 2$ such that

(3) $\forall f \in H(\beta)\left[f \subseteq u \Rightarrow \forall \eta<\kappa \exists y \in I_{\beta}(X(f)-\eta \in y)\right]$.

Proof. Let $\mathscr{U} \in \beta \kappa-\mathrm{NU}(\kappa)$ with $\mathscr{U} \in \bar{I}_{\beta}$. Define $u$ : $\beta \rightarrow 2$ by $u(\xi)=1$ iff $X_{\xi} \in \mathscr{U}$.

We now define $x_{i, \beta}(i<2)$ by

$$
x_{i, \beta}=u \cup\left(2^{\kappa}-\beta\right) \times\{i\} .
$$

Then the following truncated version of $\left(\mathrm{B}_{\beta+1}\right)$ holds:

$\left(\mathbf{B}^{\prime}\right) \forall i<2 \forall \xi \leq \beta \forall f \in H(\beta)\left[f \subseteq x_{i, \xi} \Rightarrow \forall \eta<\kappa \exists y \in I_{\xi}(X(f)-\eta \in y)\right]$.

We must construct $X_{\beta}$ in such a way that the $(\beta+1)$-sequence $\left\langle X_{\xi}: \xi<\beta+1\right\rangle$ satisfies $\left(\mathbf{A}_{\beta+1}\right)$ and $\left(\mathbf{B}_{\beta+1}\right)$. For both conditions we only have to consider functions $f \in H(\beta+1)$ with $\beta \in \operatorname{dom}(f)$, for if $\beta \notin \operatorname{dom}(f)$ then we know that the statement between square brackets is satisfied because of $\left(\mathrm{A}_{\beta}\right)$ and $\left(\mathrm{B}^{\prime}\right)$. Since $|\beta|<2^{\kappa}$, we see that we have to construct $X_{\beta}$ in such a way that less than $2^{\kappa}$ conditions on $X_{\beta}$ (or $\kappa-X_{\beta}$ ) are satisfied.

We slightly rephrase these conditions so as to emphasize the information we have from $\left(\mathbf{A}_{\beta}\right)$ and $\left(\mathbf{B}^{\prime}\right)$ :

Conditions of type $\left(\mathbf{A}_{\beta+1}\right)$. Given $f \in H(\beta)$ we know that $X(f)=\kappa$, and we want $\left|X(f) \cap X_{\beta}\right|=\left|X(f)-X_{\beta}\right|=\kappa$, or, equivalently,

$$
\forall \eta<\kappa \exists \gamma, \delta \in X(f)\left[\gamma, \delta>\eta \wedge \gamma \in X_{\beta} \wedge \delta \notin X_{\beta}\right]
$$

Conditions of type $\left(\mathbf{B}_{\beta+1}\right)$. Given $i<2, \xi \leq \beta, f \in H(\beta)$ with $f \subset x_{i, \xi}$ we know that for each $\eta<\kappa$ there is $y_{\eta} \in I_{\xi}$ with $X(f)-\eta \in y_{\eta}$. Since $y_{\eta}$ is a nonuniform ultrafilter on $\kappa$ it follows that there is $Y_{\eta} \in y_{\eta} \cap[\kappa]^{<\kappa}$ with $Y_{\eta} \subseteq X(f)-\eta$.

So it would be sufficient for our purposes to know that

$$
\forall \eta<\kappa \exists \eta^{\prime}, \eta^{\prime \prime}<\kappa\left[\eta^{\prime}, \eta^{\prime \prime} \geq \eta \wedge\left(Y_{\eta^{\prime}} \subseteq \kappa-X_{\beta}\right) \wedge\left(Y_{\eta^{\prime \prime}} \subseteq X_{\beta}\right)\right]
$$

Since then $Y_{\eta^{\prime}} \subseteq X(f \cup\{\langle\beta, 0\rangle\})$, hence $X(f \cup\{\langle\beta, 0\rangle\})-\eta \in Y_{\eta^{\prime}} \in I_{\xi}$; and $Y_{\eta^{\prime \prime}} \subseteq X(f \cup\{\langle\beta, 1\rangle\})$, so $X(f \cup\{\langle\beta, 1\rangle\})-\eta \in Y_{\eta^{\prime \prime}} \in I_{\xi}$.

If we assume $\mathrm{GCH}$, then there are $\kappa$ such conditions to meet; since $\kappa$ is regular we can find $X_{\beta}$ with an easy construction, adding in $\kappa$ steps singleton sets or elements of $[\kappa]^{<\kappa}$ to $X_{\beta}$ or $\kappa-X_{\beta}$ or both.

If we assume $\kappa=\omega$ and MA, then our conditions tell us that $X_{\beta}$ has to have infinite intersection with each member of a certain collection of less than $2^{\omega}$ infinite subsets of $\omega$, and $\omega-X_{\beta}$ also has to have infinite intersection with each member of a certain collection (not necessarily the same) of less than 
$2^{\omega}$ infinite subsets of $\omega$. (Since, in this case, we can pick each $Y_{\eta}$ to be a singleton, we take appropriate unions of the $Y_{\eta}$ 's for each pair $i, \xi$.) It is a well-known consequence of MA that this is possible (cf. Remark 7.2).

7.1. Remark. There exists in ZFC a family $\left\langle X_{\xi}: \xi<2^{\kappa}\right\rangle$ satisfying only (1) $[\mathrm{H}]$.

7.2. Remark. Since initial $\omega$-compactness is not finitely productive in ZFC, and since MA frequently makes cardinals $\kappa$ with $\omega<\kappa<\mathfrak{c}$ look like $\omega$, one may feel that Theorem 1.6 (initial $\omega_{\omega}$-compactness is not finitely productive under MA $\left.+\mathfrak{c}>\omega_{\omega}\right)$ is not too surprising, and one may wonder if there can be a singular $\kappa \geq \mathfrak{c}$ such that initial $\kappa$-compactness is not finitely productive. This turns out to be the case. We need a generalization of a weak form of MA for higher cardinals.

For a cardinal $\kappa$ consider the partial order $\mathbb{D}_{\kappa}=\left\langle\mathbb{D}_{\kappa}, \leq\right\rangle$, defined by

$$
\begin{gathered}
\mathbb{D}_{\kappa}=\left\{\langle A, B\rangle: A, B \in[\kappa]^{<\kappa} \text { are Disjoint }\right\}, \\
\langle A, B\rangle \leq\langle C, D\rangle \text { if } A \supseteq C \text { and } B \supseteq D .
\end{gathered}
$$

Consider the statement

$\Delta_{\kappa}: 2^{\lambda}=\lambda^{+}$if $\omega \leq \lambda<\kappa$, and if $\mathscr{D}$ is a collection of dense subsets of $\mathbb{D}_{\kappa}$ with $|\mathscr{D}|<2^{\kappa}$, then there is a filter on $\mathbb{D}_{\kappa}$ which intersects every member of $\mathscr{D}$.

Clearly MA implies $\Delta_{\omega}$. Baumgartner proved (more than) that it is consistent with ZFC that $\Delta_{\omega_{1}}+2^{\omega_{1}}$ is as large as you like [B, Theorem 4.2]; the same proof shows that if $\kappa$ is regular, then it is consistent with ZFC that $\Delta_{\kappa}+2^{\kappa}$ is as large as you like.

It is easy to see that our construction works under $\Delta_{\kappa}$. If there is a singular $\lambda$ with $\kappa<\lambda<2^{\kappa}$, and $2^{\kappa}$ is regular, then this yields two initially $\lambda$-compact normal spaces whose product is not initially $\lambda$-compact.

\section{Proof that $E_{0} \times E_{1}$ is Normal if $\kappa=\omega$}

Recall that we are assuming MA, so $c$ is regular.

Since the points of $\omega \times \omega$ are isolated, it suffices to prove that every two disjoint closed subsets of

$$
X=\left(E_{0} \times E_{1}\right)-(\omega \times \omega)=\left(\Sigma_{0} \times \Sigma_{1}\right) \cup\left(\omega \times \Sigma_{1}\right) \cup\left(\Sigma_{0} \times \omega\right)
$$

have disjoint closures in

$$
\bar{X}=(b \omega \times b \omega)-(\omega \times \omega)=\left({ }^{\mathfrak{c}} 2 \times{ }^{\mathfrak{c}} 2\right) \cup\left(\omega \times{ }^{\mathfrak{c}} 2\right) \cup\left({ }^{\mathfrak{c}} 2 \times \omega\right) .
$$

Recall from $\S 5$, in particular from Remark 5.4, that

(1) $\Sigma_{i}$ is $\omega$-bounded $(i<2)$;

(2) every two disjoint closed subsets of $\Sigma_{i}$ have disjoint closures in ' $2(i<$ $2)$; and

(3) every two disjoint closed subsets of $\Sigma_{0} \times \Sigma_{1}$ have disjoint closures in ' $2 \times{ }^{\prime} 2$.

Let $F$ and $G$ be any two disjoint closed subsets of $X$. Since $X$ has no isolated points we cannot simplify the argument by considering the nonisolated points only, as we did when proving that $E_{0}$ and $E_{1}$ are normal. However, we use a similar idea: we look at the more nonisolated points first. 
Claim. $\bar{F} \cap \bar{G} \cap{ }^{\mathfrak{c}} 2 \times{ }^{\mathfrak{c}} 2=\varnothing$.

Assuming that the Claim holds, it is easy to see from (2) and the above formula for $\bar{X}$ that $\bar{F} \cap \bar{G}=\varnothing$.

To prove the Claim, let $\langle a, b\rangle \in{ }^{\mathfrak{c}} 2 \times{ }^{\mathfrak{c}} 2$ be arbitrary. Then

$$
\langle a, b\rangle \notin\left(F \cap\left(\Sigma_{0} \times \Sigma_{1}\right)\right)^{-} \cap\left(G \cap\left(\Sigma_{0} \times \Sigma_{1}\right)\right)^{-},
$$

by (3). Hence we can find open $A, B$ in $b \omega$ with $a \in A, b \in B$ such that $\bar{A} \times \bar{B}$ does not intersect both $F \cap\left(\Sigma_{0} \times \Sigma_{1}\right)$ and $G \cap\left(\Sigma_{0} \times \Sigma_{1}\right)$. Without loss of generality

(4) $(\bar{A} \times \bar{B}) \cap F \cap\left(\Sigma_{0} \times \Sigma_{1}\right)=\varnothing$.

We assert that for some $n<\omega$ we have

$$
[(\bar{A}-n) \times(\bar{B}-n)] \cap F=\varnothing .
$$

Clearly this assertion implies $\langle a, b\rangle \notin \bar{F}$, hence $\langle a, b\rangle \notin \bar{F} \cap \bar{G}$.

Suppose our assertion to be false. Then there is a strictly increasing sequence $\left\langle k_{n}\right\rangle_{n}$ in $\omega$ and a sequence $\left\langle x_{n}\right\rangle_{n}$ in ' 2 such that one of the following holds:

(a) $\left\langle k_{n}, x_{n}\right\rangle \in F \cap(A \times B)$ and $x_{n} \in \Sigma_{1}$, for all $n<\omega$; or

(b) $\left\langle x_{n}, k_{n}\right\rangle \in F \cap(A \times B)$ and $x_{n} \in \Sigma_{0}$, for all $n<\omega$.

Without loss of generality we assume that (a) holds. Since $E_{0}=\omega \cup \Sigma_{0}$ is countably compact, it follows from (1) that $E_{0} \times \Sigma_{1}$ is countably compact. Hence the $\left\langle k_{n}, x_{n}\right\rangle$ 's have a cluster point $\langle s, t\rangle$ in $E_{0} \times \Sigma_{1}$. Since the $k_{n}$ 's are distinct, we have $s \in \Sigma_{0}$. This contradicts (4) since clearly $\langle s, t\rangle \in F$. This completes the proof.

8.1. Remark. Unlike before it is not true that $\beta\left(E_{0} \times E_{1}\right)=b \omega \times b \omega$, for $\{\langle n, n\rangle: n<\omega\}$ and $\Sigma_{0} \times \Sigma_{1}$ are disjoint closed subsets of $E_{0} \times E_{1}$ which do not have disjoint closures in $b \omega \times b \omega$. (Alternatively, since $E_{0} \times E_{1}$ is not pseudocompact one can apply Glicksberg's Theorem [G, Theorem 1].)

8.2. Remark. The above argument also shows that $E_{i} \times E_{i}$ is normal and that $\left(E_{i} \times E_{i}\right)-(\omega \times \omega)$ is countably compact $(i<2)$. It follows that if $E=E_{0}+E_{1}$, then $E$ is countably compact, and $E \times E$ is normal and countably paracompact but not pseudocompact $(E \times E$ is countably paracompact since its subspace of nonisolated points is countably compact). This gives a consistent counterexample to two questions: Keesling asked if all finite powers of $X$ are pseudocompact if $X$ is countably compact and normal [ $\mathrm{K}]$, and Parsons asked if $X \times X$ is countably compact if $X$ is countably compact and normal and $X \times X$ is countably paracompact [P]; Parsons gives an honest example of a countably compact $X$ such that $X \times X$ is countably paracompact but not countably compact. I am indebted to Jerry Vaughan for bringing these questions to my attention.

8.3. Remark. $X$ has two "levels": $\omega \times \Sigma_{1} \cup \Sigma_{0} \times \omega$ and $\Sigma_{0} \times \Sigma_{1}$. I was not interested in attempting the four level proof that $E_{0} \times E_{1}$ is normal for $\kappa>\omega$.

\section{REFERENCES}

[B] J. E. Baumgartner, Iterated forcing, Surveys in Set Theory (A. R. D. Mathias, ed.), Cambridge Univ. Press, 1983, pp. 1-59.

[C] H. H. Corson, Normality in subsets of product spaces, Amer. J. Math. 81 (1959), 785-796.

[vD] E. K. van Douwen, The product of two countably compact topological groups, Trans. Amer. Math. Soc. 262 (1980), 417-427. 
[FG] N. J. Fine and L. Gillman, Extension of continuous functions in $\beta N$, Bull. Amer. Math. Soc. 13 (1962), 29-36.

[Fr] Z. Frolík, Sums of ultrafilters, Bull. Amer. Math. Soc. 73 (1963), 87-91.

[G] I. Glicksberg, Stone-Čech compactifications of products, Trans. Amer. Math. Soc. 90 (1959), 369-382.

[GFW] S. L. Gulden, W. M. F. Fleischman, and J. H. Weston, Linearly ordered topological spaces, Proc. Amer. Math. Soc. 24 (1970), 197-203.

[H] F. Hausdorff, Über zwei Sätze von G. Fichtenholz und L. Kantorovich, Studia Math. 6 (1936), 18-19.

[K] J. Keesling, Normality and compactness are equivalent in hyperspaces, Bull. Amer. Math. Soc. 76 (1970), 618-619.

[Ko] A. P. Kombarav, On the normality of $\Sigma_{m}$-products, Dokl. Akad. Nauk SSSR 211 (1973), 524-527 = Soviet Math. Dokl. 14 (1973), 1050-1054.

[KoM] A. P. Kombarova and V. I. Malyhin, On $\Sigma$-products, Dokl. Akad. Nauk SSSR 211 (1973), 774-776 = Soviet Math. Dokl. 14 (1973), 1780-1783.

[Ku] K. Kunen, Set theory, North-Holland, Amsterdam, 1980.

[N] J. Novák, On the cartesian product of two compact spaces, Fund. Math. 40 (1953), 106-112.

[P] L. Parsons, An example of a space which is countably compact whose square is countably paracompact but not countably compact, Proc. Amer. Math. Soc. 65 (1977), 351-354.

[R] M. E. Rudin, Lecture notes in set-theoretic topology, Regional Conf. Ser. in Math., no. 23, Amer. Math. Soc., Providence, R.I., 1973.

[SS] V. Saks and R. M. Stephenson, Products of m-compact spaces, Proc. Amer. Math. Soc. 28 (1971), 279-328.

[SV] R. M. Stephenson and J. E. Vaughan, Products of initially m-compact spaces, Trans. Amer. Math. Soc. 196 (1974), 177-189.

[T] H. Teresaka, On cartesian product of compact spaces, Osaka J. Math. 4 (1952), 11-15.

Department of Mathematics, Ohio University, Athens, Ohio 45701 